\title{
Rotura recurrente de neovejiga ileal: manejo conservador
}

\author{
Ascaso Til H, Segarra Tomás J, De la Torre Holguera P*, Monllau Font V, Palou Redorta J, \\ Villavicencio Mavrich $\mathrm{H}$.
}

Servicio de Urología. Radiología*. Fundació Puigvert. Barcelona.

Actas Urol Esp. 2007;31(3):279-284

\section{RESUMEN}

ROTURA RECURRENTE DE NEOVEJIGA ILEAL: MANEJO CONSERVADOR

Objetivo. Exposición de un caso de rotura recurrente de neovejiga tratada de forma conservadora. Revisión de los artículos publicados desde 1985 sobre tratamiento conservador en estos casos.

Resultados. Desde 1985 se han publicado 19 artículos sobre rotura espontánea de neovejigas, de los cuales, 4 hablan de la posibilidad de tratamiento conservador con éxito, siempre que los paciente sean bien seleccionados.

Conclusiones. En pacientes con neovejiga es fundamental sospechar una perforación espontánea ante dolor abdominal, y, si es posible, confirmarlo mediante cistografía o TC. En muchas ocasiones, será necesaria una laparotomía exploradora, bien por la falta de diagnóstico, bien porque el estado del paciente no permite otra actitud, pero en casos seleccionados y con un diagnóstico preciso, el tratamiento conservador puede ser resolutivo.

Palabras clave: Rotura. Perforación. Tratamiento. Neovejiga.

\section{ABSTRACT}

\section{RECURRENT NEOBLADDER RUPTURE: CONSERVATIVE MANAGEMENT}

Purpose. To present a case of recurrent neobladder rupture treated in a conservative fashion. To review the articles on conservative management of cases like this one published from 1985.

Results. Nineteen articles have been published since 1985 on spontaneous neobladder rupture, 4 of which describe the possibility of a successful conservative management, provided that the patients are carefully selected.

Conclusions. Faced to abdominal pain in patients with neobladder, it is essential to suspect spontaneous perforation and, if possible, to confirm such suspicions by means of cystography or CT. An exploratory laparotomy may be necessary in many instances, sometimes due to lack of a diagnosis and sometimes because the patient's status rules out any other procedure, but in selected and precisely diagnosed cases a conservative management can be resolvent.

Keywords: Rupture. Perforation. Treatment. Neobladder.

$\mathrm{L}$ a cistectomía es el tratamiento de elección del cáncer vesical infiltrante. Si la localización del tumor lo permite, la sustitución vesical ortotópica con neovejigas construidas con segmentos intestinales destubularizados mejora la calidad de vida de estos pacientes, al no deteriorar su autoimagen y permitir una micción similar a la fisiológica. El coste es una mayor dificultad técnica y un mayor número de complicaciones inmediatas. La rotura espontánea de reservorios intestinales ha sido descrita principalmente en enterocistoplastias de ampliación ${ }^{1}$ y con menos frecuencia en neovejigas ileales e ileocolónicas ortotópicas.

Se expone el caso de un paciente con neovejiga ileal tipo Camey II, que presentó dos perforaciones a los 6 y 9 años de la cirugía; la primera diagnosticada y tratada por laparotomía exploradora y la segunda manejada conservadoramente. Es el primer caso publicado de perforación recurrente que no ha sido sometido a dos laparotomías para diagnóstico y tratamiento. 


\section{CASO CLÍNICO}

Paciente varón de 61 años con alergia al ácido acetilsalicílico, metamizol, y cibalgina, que en julio de 1995 es intervenido por carcinoma vesical infiltrante (estadio patológico: G3 pT3a) practicándose cistoprostatectomía radical y sustitución vesical con neovejiga ileal tipo Camey II. Evolución anodina. Al poco tiempo, el paciente no requería de autosondajes por residuo postmiccional escaso.

En julio de 2001 presenta cuadro de dolor en hipocondrio derecho, asociado a náuseas y vómitos sin fiebre, de varios días de evolución, diagnosticado de cólico hepático y manejado conservadoramente. Por persistencia del dolor se realiza ecografía y tomografía computerizada (TC) abdominal, observándose colelitiasis no complicada y presencia de líquido libre peri hepático, peri esplénico, paracólico y en pelvis. Con el diagnóstico de abdomen agudo, se realiza laparotomía exploradora, encontrando líquido seropurulento libre en cavidad peritoneal y una perforación de $5 \mathrm{~mm}$ en la pared lateral derecha de la neovejiga. Se realiza sutura de la lesión y refuerzo de la misma mediante colgajo de epiplón mayor. Postoperatorio complicado por broncoaspiración con inestabilidad hemodinámica que requiere cuidados semicríticos. Al alta se inicia programa de autocateterismos con escaso residuo postmiccional. Tres años más tarde (septiembre de 2004), el paciente presenta, poco después de levantarse por la mañana, cuadro de dolor abdominal, peritonismo e íleo paralítico, asî como disminución de la diuresis, sin fiebre. Refiere autocateterismo la noche anterior, sin dificultad, dolor ni hematuria. La analítica muestra $\mathrm{Hb} 156 \mathrm{~g} / 1$, leucocitosis de 16,2 g/1 (66,1\% segmentados) más desviación izquierda, INR 0,97, $\mathrm{Na} 141 \mathrm{mmol} / 1$, K 3,9 mmol/1, Creatinina 174 $\mathrm{mmol} / \mathrm{l}$, urea $11,1 \mathrm{mmol} / 1$. Se realiza TC abdominal que demuestra abundante líquido libre peritoneal y rotura de la neovejiga con extravasado de contraste (Fig. 1). Se coloca sonda vesical, obteniendo 800 cc de orina y se practica paracentesis diagnóstica con bioquímica compatible con orina. El cultivo resultó positivo para E. Coli, sensible a amoxicilina-clavulánico y aztreonam.

Dada la estabilidad hemodinámica del paciente y el corto tiempo de evolución del proceso, se

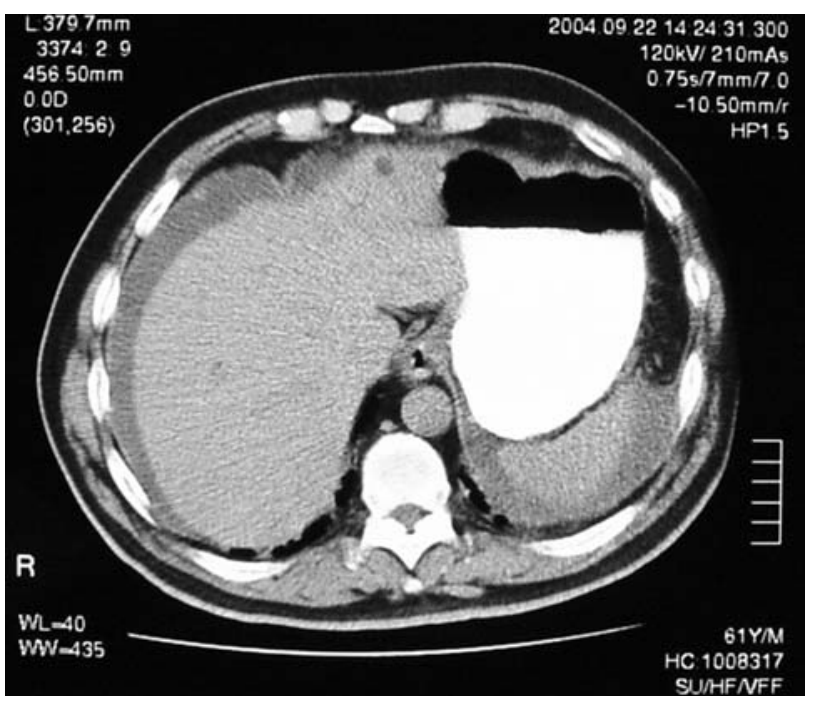

FIGURA 1A. TC abdominal. Abundante liquido libre peritoneal (perihepático, periesplénico).

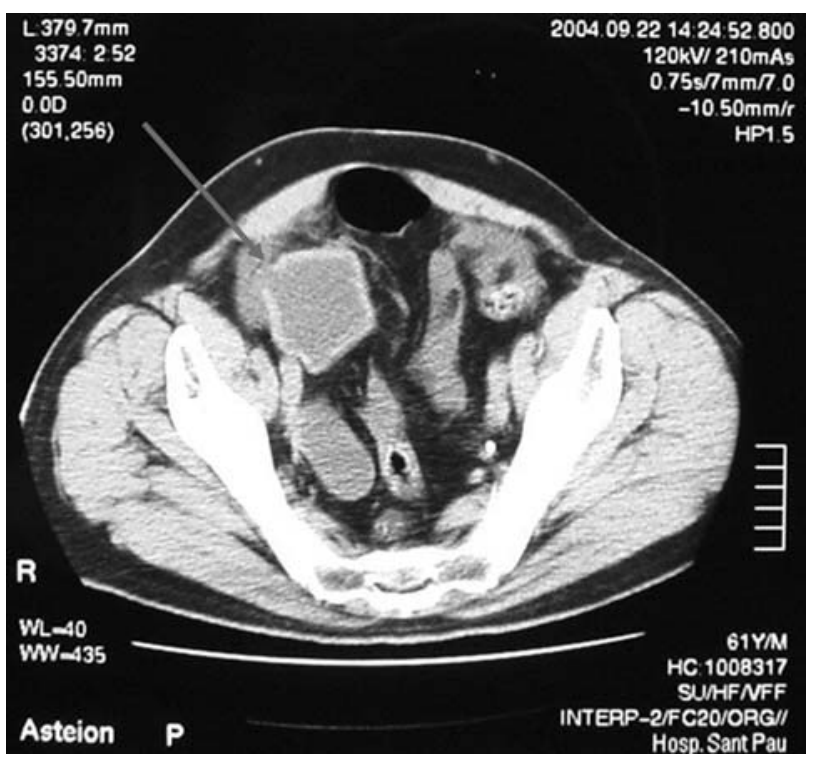

FIGURA 1B. TC abominal. Rotura de neovejiga en pared lateral derecha.

realiza tratamiento conservador, colocándose sondas de nefrostomía bilateral (SNF), drenaje peritoneal continuo con pig-tail $6 \mathrm{~F}$ y sonda vesical. Además se inicia tratamiento antibiótico de amplio espectro (amoxicilina-clavulánico $1 \mathrm{gr} / \mathrm{ev} /$ $8 \mathrm{~h}$ y aztreonam $1 \mathrm{gr} / \mathrm{ev} / 12 \mathrm{~h})$. La evolución clínica $\mathrm{y}$ analítica fue satisfactoria.

Una semana después, se realiza cistografía de relleno que informa de neovejiga de amplia capacidad, reflujo bilateral y ausencia de extravasado de contraste (Fig. 2), procediéndose a la retirada de las SNF y 7 días más tarde de la sonda vesical. 


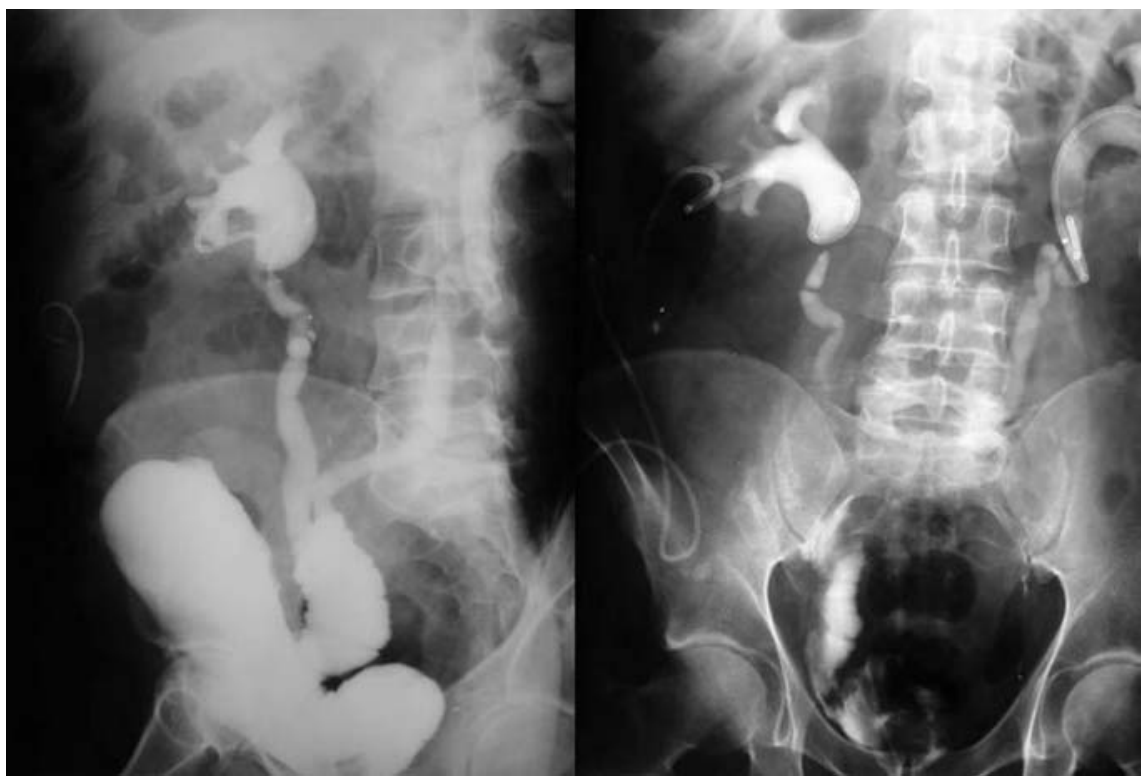

FIGURA 2. Cistografía de relleno a la semana de la rotura.

Se inicia programa de autocateterismos diarios demostrando escaso residuo postmiccional confirmado mediante ecografias seriadas.

\section{DISCUSIÓN}

La rotura espontánea de reservorios intestinales se ha descrito principalmente en enterocistoplastias de ampliación ${ }^{1}$ siendo menos frecuentes en neovejigas ileales e ileocolónicas ortotópicas. Mansson en $1997^{2}$ recopiló la experiencia escandinava sobre roturas en derivaciones cutáneas continentes y sustituciones vesicales ortotópicas, encontrando 17 pacientes con episodios de perforación de reservorios continentes cutáneos sobre 1070 cirugias (incidencia del $1,5 \%$ ) y 1 perforación de neovejiga ortotópica sobre 650 pacientes (incidencia del 0,15\%). Miller ${ }^{3}$ en su serie de 158 neovejigas ileales sólo ha tenido un caso de rotura espontánea (incidencia del 0,5\%). La baja incidencia de perforaciones en neovejigas ortotópicas de estas series contrasta con la de Nippgen ${ }^{4}$ que presenta 5 roturas en 4 pacientes sobre un total de 93 neovejigas ileales tipo Hautman (incidencia del $4,3 \%$ ) o la de Desgrandchamps ${ }^{5}$ con 5 roturas espontáneas en 103 neovejigas ileales (incidencia del 4,8\%).

Aunque la perforación es una de las complicaciones menos frecuentes de las neovejigas ortotópicas debe ser tenida en cuenta por la gravedad que puede representar su retraso diagnóstico.

\section{ETIOLOGÍA}

Se han propuesto diferentes mecanismos como causa de esta perforación ${ }^{6}$. La sobredistensión aguda o crónica de la neovejiga con compromiso vascular e isquemia es una de las causas más argumentadas, tanto clínica ${ }^{4,6-9}$ como experimentalmente ${ }^{10}$, pudiendo aparecer por incumplimiento de la recomendación de micción pautada ${ }^{4}$, por tapón mucoso uretral $^{11}$ o dificultad para cateterización. Otras causas menos probables son la presencia de adherencias intraperitoneales a la neovejiga, que pueden causar desgarros durante los episodios de sobredistensión ${ }^{1}$, o lesiones directas de la pared intestinal por mala técnica de autosondaje (tenerse en cuenta cuando hay hematuria simultánea al sondaje) ${ }^{12}$. Las líneas de sutura de la neovejiga no parecen ser un punto débil en el que se produzcan más frecuentemente roturas ${ }^{2,6,13}$.

Hay comorbilidades, que no justifican por si mismas la rotura de la neovejiga, pero predisponen a ella o empeoran la evolución de ésta una vez producida: la presencia de infección urinaria, frecuente en pacientes sometidos a autocateterismos, puede debilitar la pared intestinal ${ }^{1}$, el antecedente de radioterapia pelviana aumenta el grado de isquemia de la pared de la neovejiga $^{14}$, la diabetes mellitus interfiere en la cicatrización tisular y produce neuropatía periféri$\mathrm{ca}^{4}$, el alcoholismo predispone a la sobredistensión y traumas sobre la neovejiga ${ }^{4,9}$, la poliquimioterapia $^{11}$ favorece la sobredistensión por ingesta forzada de líquidos y altera los mecanismos defensivos.

Independientemente del mecanismo lesional, es posible una recurrencia de la perforación, como sucede en el caso que presentamos y en otros de la literatura ${ }^{4}$.

De los casos publicados, incluyendo el presente, el $64 \%$ han presentado rotura en la pared lateral derecha de la neovejiga ( 16 de las 25 perforaciones), quizás debido a que esta zona se corresponde con el íleon terminal, a $15-20 \mathrm{~cm}$. 
del ciego. Para el aislamiento de este segmento intestinal se ha de dividir la arteria ileocólica pudiendo condicionar que ésta sea la parte más isquémica de la neovejiga ${ }^{5}$ y que esta perfusión empeore más durante los episodios de sobredistensión ${ }^{15}$ (Tabla 1).

\section{DIAGNÓSTICo}

Es muy importante la sospecha de esta entidad, ya que a veces, el dolor abdominal no es tan intenso como sería esperable, debido a una percepción sensorial anómala de estas vejigas ${ }^{6}$, pudiendo demorarse el diagnóstico.

La clínica puede variar desde un abdomen distendido y levemente doloroso hasta una peritonitis franca. Es frecuente ver dificultad miccional o retención urinaria. Si el tiempo de evolución se prolonga, puede instaurarse un cuadro séptico ${ }^{1}$.

En muchas ocasiones, el diagnóstico de perforación de neovejiga se realiza durante una laparotomía exploradora por abdomen agudo, bien porque no se ha establecido la sospecha clínica, bien porque aun habiéndola establecido, las técnicas de imagen no consiguen confirmarla. Las cistografías tienen una alta tasa de falsos negativos probablemente porque el volumen de contraste infundido no logra distender adecuadamente la neovejiga o porque la lesión es de pequeño tamaño y el moco la ocluye parcialmente. Mansson ${ }^{2}$ en su revisión de reservorios continentes cutáneos realizo 6 cistografias de las cuales sólo 4 fueron diagnósticas. Nippgen ${ }^{4}$ en su serie de 5 perforaciones en neovejigas tipo Hautmann realizó cistografia en 3 ocasiones siendo diagnóstica en 2 . En la revisión de la literatura de roturas en neovejigas ortotópicas, encontramos que de los 23 casos publicados hasta la fecha, la cistografía se realizó en 12 de ellos, siendo diagnóstica en el 33,3\%, y no demostró fuga de contraste en el resto $(66,6 \%)$. Una cistografía negativa no descarta el diagnóstico de rotura de neovejiga.

La TC abdominal suele demostrar abscesos y la presencia de líquido libre intraperitoneal, pero raramente define el origen del mismo. Sólo hay un caso descrito en el que identificó la perforación de la neovejiga ${ }^{9}$. En nuestro caso también ha permitido definir claramente el lugar de la lesión de la neovejiga (Fig 1).
La cistografia por TC puede ser más sensible, pues ha permitido demostrar la fuga de contraste en el único caso publicado en el que se usó la técnica ${ }^{8}$. Pero un solo caso no es suficiente para poder afirmarlo.

\section{TRATAMIENTO}

El tratamiento de elección de la rotura espontánea de una neovejiga es la laparotomía con cierre de la lesión y drenaje del urinoma ${ }^{9,13}$. Esta agresividad terapéutica viene avalada por la dificultad diagnóstica del cuadro y la mortalidad que históricamente ha presentado. Elder, en $1988^{1}$ publicó una mortalidad del $23-25 \%$ en vejigas ampliadas que sufrieron perforación. La gravedad y rápida progresión del cuadro clínico queda patente en el caso presentado por Kyriakidis ${ }^{14}$, que falleció por una gangrena de Fournier tras rechazar la cirugía de la perforación vesical.

La laparotomía puede ser técnicamente dificil por las múltiples intervenciones abdominales previas de estos pacientes. Por ello, algunos autores recomiendan intentar tratamiento conservador no quirúrgico en pacientes con diagnóstico temprano y estabilidad hemodinámica ${ }^{16}$. Otros opinan que el manejo conservador con drenaje vesical y antibióticos es una opción cuando la orina no está infectada, mientras que si lo está o hay peritonitis, el tratamiento de elección es la laparotomía ${ }^{12}$. Baseman $^{11}$ en 1997 manejó conservadoramente dos roturas de neovejiga ileal tipo Kock, Parsons ${ }^{8}$ en el 2001 hizo lo propio con una rotura de neovejiga tipo Studer y Gupta ${ }^{17}$ fue el primero en tratar conservadoramente una perforación de neovejiga sigmoide. Tanto Parsons como Gupta definieron una serie de características que pueden ayudarnos al intentar un tratamiento conservador: diagnóstico temprano, estabilidad hemodinámica, escasa repercusión analítica, ausencia de comorbilidades que puedan complicar la evolución del cuadro, monitorización clínica y analítica estricta, drenaje adecuado de la neovejiga y las colecciones que estén presentes, reposo intestinal y tratamiento con antibioticoterapia de amplio espectro. Siempre teniendo presente que ante cualquier signo de mala evolución ha de procederse a la cirugía. La mayoría de estos supuestos se cumplían en nuestro caso, indicando tratamiento conservador con una evolución satisfactoria. 
Tabla 1

Resumen de la literatura

\begin{tabular}{|c|c|c|c|c|c|c|}
\hline Autor & $\begin{array}{l}\text { Tipo } \\
\text { neovejiga }\end{array}$ & Clinica (1) & $\begin{array}{l}\text { Drenaje de } \\
\text { orina al sondar } \\
\text { durante cuadro }\end{array}$ & $\begin{array}{l}\text { Exploración } \\
\text { diagnóstica (2) }\end{array}$ & $\begin{array}{l}\text { Causa } \\
\text { sospechada }\end{array}$ & Tratamiento (3) \\
\hline Haupt $^{15}$ & Ileal & $\mathrm{P}$ & Rao. no precisa & $\begin{array}{l}\mathrm{C}=\text { negativa } \\
\mathrm{LE}=\text { positiva }\end{array}$ & Tapón mucoso & $\mathrm{L}$ \\
\hline Miller $^{3}$ & Ileal & ¿? & ¿? & ¿? & ¿? & ¿? \\
\hline Thompson ${ }^{6}$ & Ileocolónica & P, escalofrios, afebril & $1.600 \mathrm{ml}$ turbia & $\begin{array}{l}\mathrm{C}=\text { negativa } \\
\mathrm{LE}=\text { positiva }\end{array}$ & Sobredistensión & $\mathrm{L}$ \\
\hline Martínez $^{13}$ & $\begin{array}{l}\text { Ileal } \\
\text { (Camey I) }\end{array}$ & $\begin{array}{l}\mathrm{P}, \text { vómitos, afebril } \\
\mathrm{L}=14.000\end{array}$ & $\begin{array}{l}\text { RAO por } \\
\text { coágulos }\end{array}$ & $\mathrm{C}=$ positiva & $\begin{array}{l}\text { Coágulos y moco que obligan } \\
\text { a autocateterismo y tras éste } \\
\text { aparece el cuadro bruscamente. }\end{array}$ & $\mathrm{L}$ \\
\hline Watanabe $^{18}$ & Ileal & $\begin{array}{l}\text { Dolor abdominal bajo } \\
\text { escalofrios y fiebre } \\
\mathrm{A} / \mathrm{O}=\text { neg. }\end{array}$ & ¿? & $\begin{array}{l}\mathrm{C}=\text { negativa } \\
\mathrm{LE}=\text { positiva }\end{array}$ & Adherencias al colon sigmoide & $\mathrm{L}$ \\
\hline $\begin{array}{l}\text { Baseman }^{11} \\
2 \text { casos }\end{array}$ & Ileal (Kock) & $\begin{array}{l}\text { Dolor abdominal, } \\
\text { afebril } \\
\mathrm{A} / \mathrm{O}=\text { neg } \\
\mathrm{L}=\text { normal }\end{array}$ & $\begin{array}{l}\text { Caso } 1=150 \mathrm{ml} \\
\text { Caso } 2=\gtreqless ?\end{array}$ & $\mathrm{C}=$ positiva & $\begin{array}{l}\text { 1/sobredistensión } \\
2 / \text { sobredistensión y trauma. }\end{array}$ & $\begin{array}{l}\text { Conservador: S/V y } \\
\text { antibióticos. }\end{array}$ \\
\hline $\begin{array}{l}\text { Desgrandchamps }{ }^{5} \\
5 \text { casos }\end{array}$ & $\begin{array}{l}\text { Ileal }(4 \text { en } \\
\text { formade "V" } \\
\text { y } 1 \text { de "W" }\end{array}$ & $\begin{array}{l}\mathrm{P} \\
\mathrm{A} / \mathrm{O}=\text { positivo en } 4 \\
\text { casos }\end{array}$ & $\begin{array}{l}\text { En } 3 \text { casos } \\
500 \mathrm{ml}\end{array}$ & $\begin{array}{l}\mathrm{C}(2 \text { casos })=\text { negativo } \\
\mathrm{LE}=\text { positiva }\end{array}$ & $\begin{array}{l}1 \text { tapón moco } \\
1 \text { tras anestesia } \\
3 \text { sobredistensión }\end{array}$ & $\mathrm{L}$ \\
\hline Ditonno $^{12}$ & Ileal & $\begin{array}{l}\text { Dolor abdominal y } \\
\text { anuria que } \\
\text { evoluciona a P }\end{array}$ & $1.000 \mathrm{ml}$ & $\begin{array}{l}\text { TAC= negativo, sólo } \\
\text { demuestra liquido } \\
\text { abdominal. } \\
\mathrm{LE}=\text { positiva }\end{array}$ & Sobredistensión & $\mathrm{L}$ \\
\hline Chen $^{19}$ & $\begin{array}{l}\text { Ileal (Camey } \\
\text { modificado) }\end{array}$ & $\begin{array}{l}\text { P y dificultad } \\
\text { miccional }\end{array}$ & $250 \mathrm{ml}$ & $\begin{array}{l}\text { CISTO= negativa } \\
\text { TAC }=\text { positivo }\end{array}$ & $\begin{array}{l}\text { Desconocido ¿sobredistensión } \\
\text { por obstrucción por moco? }\end{array}$ & $\mathrm{L}$ \\
\hline Parsons $^{8}$ & $\begin{array}{l}\text { Ileal } \\
\text { (Studer) }\end{array}$ & $\begin{array}{l}\text { P y } 38^{\circ} \mathrm{C} \\
\mathrm{L}=16.000\end{array}$ & $\begin{array}{l}\text { RAO No } \\
\text { posible sondar }\end{array}$ & $\begin{array}{l}\text { TAC= negativo. } \\
\text { Distensión vesical } \\
\text { y liquido libre peritoneo } \\
\text { CISTO= negativa para } \\
\text { perforación. } \\
\text { Estenosis de anastomosis } \\
\text { que se dilata y deja sonda } \\
\text { CISTO por TAC= positiva }\end{array}$ & Sobredistensión & $\begin{array}{l}\text { Conservador: drenaje } \\
\text { neovejiga, } \\
\text { antibióticos y } \\
\text { reposo intestinal }\end{array}$ \\
\hline Takenaka $^{9}$ & Ileal tipo "N" & $\begin{array}{l}\text { Distensión y dolor } \\
\text { abdominal + flemón } \\
\text { abdominal } \\
\mathrm{L}=24.000 \\
\mathrm{U}=103 \mathrm{C}=3,4\end{array}$ & $1.100 \mathrm{ml}$ & $\begin{array}{l}\mathrm{C}=\text { negativa } \\
\mathrm{TAC}=\text { positiva } \\
\text { Es el primer caso en } \\
\text { que el TAC hace el } \\
\text { diagnóstico }\end{array}$ & Sobredistensión & $\begin{array}{l}\text { Conservador con } \\
\text { drenaje neovejiga }\end{array}$ \\
\hline $\begin{array}{l}\text { Nippgen }{ }^{4} \text { ( } 4 \text { casos } \\
\text { con } 5 \text { perforaciones) }\end{array}$ & $\begin{array}{l}4 \text { ileales } \\
\text { (Hautmann) }\end{array}$ & $\begin{array}{l}\text { Dolor abdominal } \\
\text { severo } \\
\text { *1 paciente alcohólico, } \\
1 \text { PQT, 1DM, } \\
1 \text { obstrucción uretral }\end{array}$ & & $\begin{array}{l}\mathrm{C}=\text { Hecha en } 3 / 5 \text { casos } \\
\text { y positiva en } 2 .\end{array}$ & $\begin{array}{l}\text { Sobredistensión en } 4 \text { de los } 5 \\
\text { casos. En dos casos se añade } \\
\text { trauma y oclusión uretral. }\end{array}$ & L en todos los casos \\
\hline Gupta $^{17}$ & Sigmoide & $\begin{array}{l}\text { Distensión abdominal, } \\
\text { ileo, no peritonismo y } \\
\text { anuria de } 48 \mathrm{~h} \text {. } \\
\mathrm{A} / 0 \text { y liq. peritoneal } \\
\text { =neg. A/S aumento urea } \\
120 \mathrm{ml} / \mathrm{dl} \text { y creat. } \\
2 \mathrm{mg} / \mathrm{dl} \text { en sangre }\end{array}$ & $\begin{array}{l}\text { Anuria (vejiga } \\
\text { vacía en ECO) }\end{array}$ & $\begin{array}{l}\text { C= positiva } \\
\text { CISTO= negativa para } \\
\text { perforación. } \\
\text { Estenosis cervical que } \\
\text { se dilata } \\
\text { ECO: colección abdomen } \\
\text { y vejiga vacía }\end{array}$ & ¿? & $\begin{array}{l}\text { Conservador: sonda } \\
\text { y drenaje peritoneal. }\end{array}$ \\
\hline
\end{tabular}

(1) $\mathrm{P}=$ peritonitis/L= leucocitos en sangre. $\mathrm{A} / \mathrm{O}=$ cultivo orina. $\mathrm{U}=$ urea en $\mathrm{mg} / \mathrm{dl}$. $\mathrm{C}=$ creatinina en $\mathrm{mg} / \mathrm{dl}$.

(2) $\mathrm{C}=$ cistografia. $\mathrm{LE}=$ laparotomía exploradora. $\mathrm{TAC}=$ tomografia axial computerizada. $\mathrm{CISTO}=$ cistoscopia. $\mathrm{ECO}=$ ecografia.

(3) L= laparotomía, cierre de la lesión y drenaje vesical. 
De la experiencia de la literatura y de la extraída en nuestro caso creemos que con una adecuada selección de pacientes es posible un tratamiento conservador de esta complicación.

\section{CONCLUSIONES}

Las mejoras técnicas y la experiencia acumulada, junto con un entorno social orientado, cada día más, hacia la calidad de vida del paciente, harán que el numero de neovejigas realizadas aumente y también sus complicaciones tardías. La perforación de la neovejiga es una complicación rara pero potencialmente mortal. Dado que uno de los factores más frecuentemente asociado a la perforación es la sobredistensión de la neovejiga, es necesaria la educación de los pacientes para evitarla. Se recomienda una micción pautada independientemente de la sensación miccional, que es anómala en estos pacientes, y control del residuo postmiccional regularmente, bien con autocateterismos o ecográficamente ${ }^{5,6}$.

Ante un paciente que es portador de una neovejiga y que presenta dolor abdominal, es fundamental sospechar una perforación espontánea de la misma ${ }^{6,15}$, y, si es posible, confirmarlo con cistografia o TC. En muchas ocasiones, será necesaria una laparotomía exploradora, bien por la falta de diagnóstico, bien porque el estado del paciente no permite otra actitud, pero, en casos seleccionados con un diagnóstico preciso, el tratamiento conservador puede ser resolutivo.

\section{REFERENCIAS}

1. Elder JS, Snyder HM, Hulbert WC, Duckett JW. Perforation of the augmented bladder in patients undergoing clean intermittent catheterization. J Urol. 1988;140(5 Pt 2):1159-1162.

2. Mansson W, Bakke A, Bergman B, Brekkan E, Jonsson O, Kihl B, et al. Perforation of continent urinary reservoirs. Scandinavian experience. Scand J Urol Nephrol. 1997;31 (6):529-532.

3. Miller K, Wenderoth UK, de Petriconi R, Kleinschmidt K, Hautmann R. The ileal neobladder. Operative technique and results. Urol Clin North Am. 1991;18(4):623-630.

4. Nippgen JB, Hakenberg OW, Manseck A, Wirth MP. Spontaneous late rupture of orthotopic detubularized ileal neobladders: report of five cases. Urology. 2001;58(1):43-46.
5. Desgrandchamps F, Cariou G, Barthelemy Y, Boyer C, Teillac P, Le Duc A. Spontaneous rupture of orthotopic detubularized ileal bladder replacement: report of 5 cases. J Urol. 1997;58(3 Pt 1):798-800.

6. Thompson ST, Kursh ED. Delayed spontaneous rupture of an ileocolonic neobladder. J Urol. 1992;148(6):1890-1891.

7. Crane JM, Scherz HS, Billman GF, Kaplan GW. Ischemic necrosis: a hypothesis to explain the pathogenesis of spontaneously ruptured enterocystoplasty. J Urol. 1991;146(1): 141-144.

8. Parsons JK, Schoenberg MP. Successful conservative management of perforated ileal neobladder. J Urol. 2001; 165(4):1214-1215.

9. Takenaka A, Yamada Y, Tanaka K, Minami R, Yamanaka N. Lower abdominal phlegmon due to spontaneous rupture of an ileal neobladder. Int J Urol. 2001;8(2):75-77.

10. Essig KA, Sheldon CA, Brandt MT, Wacksman J, Silverman DG. Elevated intravesical pressure causes arterial hypoperfusion in canine colocystoplasty: a fluorometric assessment. J Urol. 1991;146(2 ( Pt 2):551-553.

11. Baseman AG, Young RR Jr, Young AK, Lerner SP. Conservative management of spontaneous rupture of Kock orthotopic ileal reservoir. Urology. 1997;49(4): 629-631.

12. Ditonno P, Battaglia M, Carrieri G, Zizzi V, Selvaggi, FP. Spontaneous rupture of an ileal neobladder 6 years after construction. J Urol. 1997;157(5):1841.

13. Martinez Jabaloyas JM, Vera Donoso CD, Morera Martinez JF, Ruiz Cerda JL, Beamud Gomez A, Jimenez Cruz, JF. Spontaneous rupture of a neobladder. Eur Urol. 1994;25 (3):259-261.

14. Kyriakidis A. Fournier's gangrene following delayed rupture of an ileal neobladder (Hautmann). Br J Urol. 1995;76(5): 668.

15. Haupt G, Pannek J, Knopf HJ, Schulze H, Senge T. Rupture of ileal neobladder due to urethral obstruction by mucous plug. J Urol. 1990. 144(3):740-741.

16. Slaton JW, Kropp KA. Conservative management of suspected bladder rupture after augmentation enterocystoplasty. J Urol. 1994;152(2 Pt 2)713-715.

17. Gupta NP, Nabi G, Ansari MS. Conservative management of spontaneous rupture of a sigmoid colon neobladder. Urol Int. 2002;69(4):325-326.

18. Watanabe K, Kato H, Misawa K, Ogawa A. Spontaneous perforation of an ileal neobladder. Br J Urol. 1994;73(4): 460-461.

19. Chen YC, Lee YH, Huang JK. Spontaneous perforation of a modified Camey neobladder. Urol Int. 1997;59(1):48-49.

Correspondencia: Dra. H. Ascaso Til Servicio de Urología. Fundació Puigvert.

Cartagena 340-350. 08025 Barcelona. Tel.: 934169700

E-mail autor: hascasotil@yahoo.es

Información artículo: Nota clínica

Trabajo recibido: diciembre 2005

Trabajo aceptado: marzo 2006 